\title{
Exact Solutions for $(3+1)$-Dimensional Potential-YTSF Equation and Discrete Kadomtsev-Petviashvili Equation
}

\author{
Yan Wang ${ }^{1,2}$ and Zhenhui Wang ${ }^{3}$ \\ ${ }^{1}$ Department of Mathematics, Shanghai University, Shanghai 200444, China \\ ${ }^{2}$ Department of Mathematics, Luoyang Normal University, Luoyang 471022, China \\ ${ }^{3}$ School of Mathematics and Information Science, Henan Polytechnic University, Jiaozuo 454003, China
}

Correspondence should be addressed to Yan Wang; wangyanshiyuan@163.com

Received 4 July 2013; Revised 28 October 2013; Accepted 11 November 2013

Academic Editor: Nazim Idrisoglu Mahmudov

Copyright (c) 2013 Y. Wang and Z. Wang. This is an open access article distributed under the Creative Commons Attribution License, which permits unrestricted use, distribution, and reproduction in any medium, provided the original work is properly cited.

By employing Hirota bilinear method, we mainly discuss the (3+1)-dimensional potential-YTSF equation and discrete KP equation. For the former, we use the linear superposition principle to get its $N$ exponential wave solutions. In virtue of some Riemann theta function formulas, we also construct its quasiperiodic solutions and analyze the asymptotic properties of these solutions. For the latter, by using certain variable transformations and identities of the theta functions, we explicitly investigate its periodic waves solutions in terms of one-theta function and two-theta functions.

\section{Introduction}

There has been considerable interest in seeking exact solutions to nonlinear differential or discrete equations. Since the exact solutions can help the physicists to well understand the mechanism of the complicated physical phenomena and dynamic processes modeled by these equations. In recent years, various approaches for constructing exact solutions are currently available such as inverse scattering transformation [1], Hirota direct method [2], the Bäcklund transformation [3], and algebra-geometric method [4-6].

Among vast exact solutions, linear superposition principle [7] and periodic or quasiperiodic wave solution [8] play a significant role in explaining the physical applications of these systems. The former method can be applied to exponential traveling waves of Hirota bilinear equations and is helpful in generating $\mathrm{N}$-wave solutions to soliton equations, particularly those in higher dimensions. The latter is also called algebra-geometric solutions or finite gap solution; it is often obtained based on the inverse spectral theory and algebrageometric method. The algebra-geometric theory, however, needs Lax pairs and is also involved in complicated analysis procedures on the Riemann surfaces. It is rather difficult to directly determine the characteristic parameters of waves, such as frequencies and phase shifts, for a function with given wave numbers and amplitudes. Based on the Hirota forms, Nakamura proposed a convenient way to find a kind of explicit quasiperiodic solution of nonlinear equations $[9,10]$; it does not need any Lax pair and Riemann surface for the given nonlinear equation and is also able to find the explicit construction of multiperiodic wave solutions. Recently, Fan et al. [11-13] extended this method and gave a uniform approach to establish the periodic solutions of nonlinear differential and difference equations. However, there are only a few works $[14,15]$ available for constructing multiperiodic wave solutions of discrete equations, since more constraint equations need to be satisfied, but the parameters in the bilinear form of these discrete equations are insufficient; thus, it is difficult to construct multiperiodic wave solutions, even for twoperiodic wave solution.

In this paper, we will mainly consider $3+1$-dimensional potential-YTSF equation and discrete KP equation. First, we use a linear superposition principle to generate $\mathrm{N}$-wave solutions of the former then use the above discussing method to construct quasiperiodic or periodic wave solutions of these two equations. In an appropriate limiting procedure, the soliton solutions are also obtained from the quasiperiodic solutions. 


\section{2. $(3+1)$-Dimensional Potential-YTSF Equation}

$(3+1)$-Dimensional potential-YTSF equation, first introduced by Yu et al. [16], may be written as

$$
-4_{x t}+u_{x x x z}+4 u_{x} u_{x z}+2 u_{x x} u_{z}+3 u_{y y}=0 .
$$

The exact solitary-wave and periodic solutions have been addressed by means of the auto-Bäcklund transformation [17], the generalized projective Riccati equation method [18], the extended homoclinic test technique [19], and so on. Using the dependent variable transformation

$$
\zeta=x+d z, \quad u=2(\ln f)_{\zeta}
$$

we transform (1) to the bilinear form

$$
\left[-4 D_{\zeta} D_{t}+d D_{\zeta}^{4}+3 D_{y}^{2}+c\right] f \cdot f=0
$$

where $d$ is an arbitrary positive constant and Hirota bilinear operators $D_{\zeta}, D_{y}$, and $D_{t}$ are defined by

$$
\begin{aligned}
D_{\zeta}^{m} D_{t}^{m^{\prime}} & f(\zeta, t) \cdot g(\zeta, t) \\
& =\left.\left(\partial_{\zeta}-\partial_{\zeta^{\prime}}\right)^{m}\left(\partial_{t}-\partial_{t^{\prime}}\right)^{m^{\prime}}\right|_{\zeta^{\prime}=\zeta, t^{\prime}=t,}
\end{aligned}
$$

which have a nice property when acting on exponential functions

$$
D_{\zeta}^{m} D_{t}^{m^{\prime}} e^{\eta_{1}} \cdot e^{\eta_{2}}=\left(k_{1}-k_{2}\right)^{m}\left(w_{1}-w_{2}\right)^{m^{\prime}} \cdot e^{\eta_{1}+\eta_{2}},
$$

with $\eta_{j}=k_{j} \zeta+l_{j} y+w_{j} t, j=1,2$.

2.1. NExponential Wave Solutions. Let us consider the special case $c=0$ in (3) and introduce $\mathrm{N}$-wave testing function

$$
f=\sum_{j=1}^{N} \epsilon_{j} f_{j}=\sum_{j=1}^{N} \epsilon_{j} e^{k_{j} \zeta+l_{j} y+w_{j} t}, \quad 1 \leq j \leq N,
$$

where $\varepsilon_{j}, k_{j}, l_{j}$, and $w_{j}$ are arbitrary constants. Substituting (6) into (3) and using (5) give

$$
\begin{gathered}
\sum_{j=1}^{N} \sum_{j=1}^{N}\left[-4\left(k_{i}-k_{j}\right)\left(w_{i}-w_{j}\right)+d\left(k_{i}-k_{j}\right)^{4}\right. \\
\left.+3\left(l_{i}-l_{j}\right)^{2}\right] f_{i} \cdot f_{j}=0 .
\end{gathered}
$$

If the following condition

$$
\begin{aligned}
& {\left[-4\left(k_{i}-k_{j}\right)\left(w_{i}-w_{j}\right)+d\left(k_{i}-k_{j}\right)^{4}+3\left(l_{i}-l_{j}\right)^{2}\right] f_{i}} \\
& \cdot f_{j}=0
\end{aligned}
$$

is satisfied, then any linear combination of the $N$ exponential wave solutions $e^{\eta_{j}}, 1 \leq j \leq N$ solves (3). By inspection, a solution to (8) is

$$
w_{j}=d k_{j}^{3}, \quad l_{j}= \pm \sqrt{d} k_{j}^{2}, \quad 1 \leq j \leq N
$$

Therefore, (1) has the following N-wave solution

$$
\begin{gathered}
u=2(\ln f)_{\zeta} \\
f=\sum_{j=1}^{N} \epsilon_{j} f_{j}=\sum_{j=1}^{N} \epsilon_{j} e^{k_{j}(x+d z) \pm \sqrt{d} k_{j}^{2} y+d k_{j}^{3} t},
\end{gathered}
$$

$$
1 \leq j \leq N,
$$

where $\epsilon_{j}, k_{j}$ are arbitrary constants. Especially assume that $\epsilon_{1}=\epsilon_{2}=\epsilon_{3}=1, k_{1}=-k_{2}$, and $k_{3}=0$ in (10); then, we get

$$
\begin{gathered}
f=1+\left(e^{k_{1}(x+d z)+d k_{1}^{3} t}+e^{-k_{1}(x+d z)-d k_{1}^{3} t}\right) e^{ \pm \sqrt{d} k_{1}^{2} y} \\
u=2(\ln f)_{\zeta}=\frac{2 k_{1} \sinh \left(k_{1}(x+d z)+d k_{1}^{3} t\right) e^{ \pm \sqrt{d} k_{1}^{2} y}}{(1 / 2)+\cosh \left(k_{1}(x+d z)+d k_{1}^{3} t\right) e^{ \pm \sqrt{d} k_{1}^{2} y}} .
\end{gathered}
$$

2.2. Quasiperiodic Solutions. The Riemann theta functions of genus one [20] are defined by

$$
\begin{aligned}
& \Theta_{1}(\xi, \tau)=-i \sum_{m=-\infty}^{\infty}(-1)^{m} e^{\pi i \xi(2 m+1)+\pi i \tau(m+(1 / 2))^{2}}, \\
& \Theta_{2}(\xi, \tau)=\sum_{m=-\infty}^{\infty} e^{\pi i \xi(2 m+1)+\pi i \tau(m+(1 / 2))^{2}}, \\
& \Theta_{3}(\xi, \tau)=\sum_{m=-\infty}^{\infty} e^{2 m \pi i \xi+\pi i \tau m^{2}}, \\
& \Theta_{4}(\xi, \tau)=\sum_{m=-\infty}^{\infty}(-1)^{m} e^{2 m \pi i \xi+\pi i \tau m^{2}},
\end{aligned}
$$

respectively, where $\xi=k \zeta+l y+w t$. Letting $\Theta_{k}(x)=\Theta_{k}(x, \tau)$ $(k=1,2,3,4)$ and using the identities

$$
\begin{aligned}
\Theta_{3}(x & +y) \Theta_{3}(x-y) \Theta_{2}^{2}(0) \\
& =\Theta_{3}^{2}(x) \Theta_{2}^{2}(y)+\Theta_{4}^{2}(x) \Theta_{1}^{2}(y), \\
\Theta_{4}(x+y) \Theta_{4}(x-y) \Theta_{2}^{2}(0) & \\
= & \Theta_{4}^{2}(x) \Theta_{2}^{2}(y)+\Theta_{3}^{2}(x) \Theta_{1}^{2}(y),
\end{aligned}
$$

we can obtain the Hirota derivatives of $\Theta_{3}(x), \Theta_{4}(x)$ :

$$
\begin{aligned}
D_{x}^{2} \Theta_{3}(x) \cdot \Theta_{3}(x) & =\left.\partial_{y}^{2} \Theta_{3}(x+y) \Theta_{3}(x-y)\right|_{y=0} \\
& =b_{1} \Theta_{4}^{2}(x)+b_{2} \Theta_{3}^{2}(x), \\
D_{x}^{4} \Theta_{3}(x) \cdot \Theta_{3}(x) & =\left.\partial_{y}^{4} \Theta_{3}(x+y) \Theta_{3}(x-y)\right|_{y=0} \\
& =c_{1} \Theta_{4}^{2}(x)+c_{2} \Theta_{3}^{2}(x), \\
D_{x}^{2} \Theta_{4}(x) \cdot \Theta_{4}(x) & =\left.\partial_{y}^{2} \Theta_{4}(x+y) \Theta_{4}(x-y)\right|_{y=0} \\
& =b_{2} \Theta_{4}^{2}(x)-b_{1} \Theta_{3}^{2}(x), \\
D_{x}^{4} \Theta_{4}(x) \cdot \Theta_{4}(x) & =\left.\partial_{y}^{4} \Theta_{3}(x+y) \Theta_{3}(x-y)\right|_{y=0} \\
& =c_{2} \Theta_{4}^{2}(x)-c_{1} \Theta_{3}^{2}(x),
\end{aligned}
$$


where

$$
\begin{gathered}
b_{1}=2 \Theta_{3}^{2}(0) \Theta_{4}^{2}(0), \quad b_{2}=2 \frac{\Theta_{2}^{\prime \prime}(0)}{\Theta_{2}(0)} \\
b_{3}=2\left(\frac{\Theta_{3}^{\prime \prime}(0)}{\Theta_{3}(0)}+\frac{\Theta_{4}^{\prime \prime}(0)}{\Theta_{4}(0)}\right), \quad b_{4}=2 \frac{\Theta_{2}^{(4)}(0)}{\Theta_{2}(0)} \\
c_{1}=2 b_{1}\left(b_{2}+b_{3}\right), \quad c_{2}=\frac{3}{2} b_{2}^{2}+b_{4} .
\end{gathered}
$$

Next, we will calculate analytically the quasiperiodic solutions using the Hirota derivatives in (14).

Case 1. In the bilinear equation (3), we suppose $f(x, y, z, t)$ to be

$$
f(x, y, z, t)=\Theta_{3}(\xi), \quad \xi=k_{1} \zeta+l_{1} y+w_{1} t
$$

where $k_{1}, l_{1}$, and $w_{1}$ are arbitrary constants. Substituting (16) into (3) and setting the coefficients of the terms $\Theta_{3}^{2}(\xi), \Theta_{4}^{2}(\xi)$ to be zero, we obtain a system of algebraic equations

$$
\begin{gathered}
\left(-4 k_{1} w_{1}+3 l_{1}^{2}\right) b_{2}+d k_{1}^{4} c_{2}+c=0 \\
\left(4 k_{1} w_{1}-3 l_{1}^{2}\right) b_{1}-d k_{1}^{4} c_{1}=0 .
\end{gathered}
$$

Solving (17), we find

$$
\begin{gathered}
w_{1}=\frac{3 l_{1}^{2} b_{1}+d k_{1}^{4} c_{1}}{4 k_{1} b_{1}}, \\
c=-\left(3 l_{1}^{2} b_{2}+d k_{1}^{4} c_{1}\right)+\frac{b_{2}}{b_{1}}\left(3 l_{1}^{2} b_{1}+d k_{1}^{4} c_{1}\right),
\end{gathered}
$$

where $b_{1}, b_{2}$ and $c_{1}, c_{2}$ are given by (15). We have thus constructed a kind of quasiperiodic solution for (1)

$$
u=2 \ln \left(\Theta_{3}\left(k_{1} \zeta+l_{1} z+w_{1} t\right)\right)_{\zeta}
$$

In the following, we further analyze the asymptotic property of (19).

Proposition 1. If the vector $\left(w_{1}, c\right)^{T}$ is given by (18) and supposing $\xi^{\prime}=2 \pi i \xi+\pi i \tau, k^{\prime}=2 \pi i k$, and $\operatorname{Im} \tau \rightarrow \infty$, then one has

$$
\begin{aligned}
f= & \Theta_{3}(\xi)=1+e^{\pi i \tau}\left[e^{2 \pi i \xi}+e^{-2 \pi i \xi}\right] \\
& +e^{4 \pi i \tau}\left[e^{4 \pi i \xi}+e^{-4 \pi i \xi}\right]+\cdots \\
= & 1+e^{\xi^{\prime}}+e^{2 \pi i \tau} e^{-\xi^{\prime}}+e^{2 \pi i \tau} e^{2 \xi^{\prime}} \cdots \\
\approx & 1+e^{\xi^{\prime}}, \quad \text { as } \operatorname{Im} \tau \rightarrow \infty .
\end{aligned}
$$

From (2), one gets the solution of (1) as follows:

$$
u=2(\ln f)_{\zeta} \longrightarrow \frac{4 k \pi i e^{\xi^{\prime}}}{1+e^{\xi^{\prime}}}=k^{\prime} \sec \frac{\xi^{\prime}}{2} e^{\left(\xi^{\prime} / 2\right)}
$$

Case 2. Assume $f(x, y, z, t)$ in (3) to be

$$
f(x, y, z, t)=\Theta_{4}(\xi), \quad \xi=k_{2} \zeta+l_{2} y+w_{2} t,
$$

where $k_{1}, l_{1}$, and $w_{1}$ are arbitrary constants. Setting the coefficients of the terms $\Theta_{3}^{2}(\xi), \Theta_{4}^{2}(\xi)$ to be zero, we have

$$
\begin{gathered}
\left(4 k_{2} w_{2}-3 l_{2}^{2}\right) b_{2}-d k_{2}^{4} c_{1}=0, \\
\left(-4 k_{2} w_{2}+3 l_{2}^{2}\right) b_{2}+d k_{2}^{4} c_{2}+c=0 .
\end{gathered}
$$

Solving (23) yields

$$
\begin{aligned}
w_{2} & =\frac{3 l_{2}^{2} b_{1}+d k_{2}^{4} c_{1}}{4 k_{2} b_{1}}, \\
c & =-\left(3 l_{2}^{2} b_{2}+d k_{2}^{4} c_{1}\right)+\frac{b_{2}}{b_{1}}\left(3 l_{2}^{2} b_{1}+d k_{2}^{4} c_{1}\right),
\end{aligned}
$$

where $b_{1}, b_{2}$, and $c_{1}, c_{2}$ are given by (15). Thus, the second kind of quasiperiodic solution of (1) is constructed as follows:

$$
u=2 \ln \left(\Theta_{4}\left(k_{2} \zeta+l_{2} z+w_{2} t\right)\right)_{\zeta}
$$

Proposition 2. If the vector $\left(w_{2}, c\right)^{T}$ is given by (24) and supposing $\xi^{\prime}=2 \pi i \xi+\pi i \tau, k^{\prime}=2 \pi i k$, and $\operatorname{Im} \tau \rightarrow \infty$, then one has

$$
\begin{aligned}
f= & \Theta_{4}(\xi)=1-e^{\pi i \tau}\left[e^{2 \pi i \xi}+e^{-2 \pi i \xi}\right] \\
& +e^{4 \pi i \tau}\left[e^{4 \pi i \xi}+e^{-4 \pi i \xi}\right]+\cdots \\
= & 1-e^{\xi^{\prime}}-e^{2 \pi i \tau} e^{-\xi^{\prime}}+e^{2 \pi i \tau} e^{2 \xi^{\prime}} \cdots \\
\approx & 1-e^{\xi^{\prime}}, \quad \text { as } \operatorname{Im} \tau \longrightarrow \infty .
\end{aligned}
$$

Using the variable transformation (2), one gets the solution of (1) as follows:

$$
u=2(\ln f)_{\zeta} \longrightarrow-\frac{4 k \pi i e^{\xi^{\prime}}}{1-e^{\xi^{\prime}}}=-k^{\prime} \csc \frac{\xi^{\prime}}{2} e^{\left(\xi^{\prime} / 2\right)} .
$$

\section{Discrete KP Equation}

The discrete analogue of KP equation [21, 22] (or a twodimensional analogue of the discrete $\mathrm{KdV}$ equation) is

$$
\nabla_{t} \frac{u+v}{1+u}=\chi \nabla_{x} E_{+y} u, \quad \nabla_{y} \frac{u+v}{1+u}=\frac{E_{+y} v-E_{-x} E_{+t} v}{\delta y\left(1+E_{+y} u\right)}
$$

where $u$ and $v$ are functions of $x, y$, and $t, \chi$ is a constant, $\nabla$ and $E$ are the difference and shift operators defined by

$$
\begin{gathered}
\nabla_{z} f(z)=\frac{1}{\delta z}\left[f\left(z+\frac{\delta z}{2}\right)-f\left(z-\frac{\delta z}{2}\right)\right] \\
E_{ \pm z} f(z)=f\left(z \pm \frac{\delta z}{2}\right) .
\end{gathered}
$$


In fact, using the dependent variable transformation

$$
\begin{gathered}
n=a\left(\frac{x}{\delta x}+\frac{y}{\delta y}\right), \\
l=b\left(\frac{y}{\delta y}+\frac{t}{\delta t}\right), \\
m=-c\left(\frac{x}{\delta x}+\frac{t}{\delta t}\right), \\
u=\frac{\zeta_{n, l}^{m} \zeta_{n, l+b}^{m+c}}{\zeta_{n, l+b}^{m} \zeta_{n, l}^{m+c}}-1, \\
v=1-\frac{\zeta_{n, l}^{m} \zeta_{n+a, l+b}^{m+c}}{\zeta_{n+a, l+b}^{m} \zeta_{n, l}^{m+c}}, \\
\chi=\frac{a(b-c)}{b(c-a)} \frac{\delta x}{\delta t}
\end{gathered}
$$

and substituting (30) into (28), we have the bilinear form of discrete KP equation as follows:

$$
\begin{gathered}
a(b-c) \zeta_{n+a, l}^{m} \zeta_{n, l+b}^{m+c}+b(c-a) \zeta_{n, l+b}^{m} \zeta_{n+a, l}^{m+c} \\
+c(a-b) \zeta_{n, l}^{m+c} \zeta_{n+a, l+b}^{m}=0
\end{gathered}
$$

where $a, b$, and $c$ are the difference intervals for independent variables $n, l$, and $m$, respectively. Some operator solutions of (31) have been addressed by the transformation groups methods $[23,24]$. This famous three-dimensional difference equation is interesting and important because it is emerging in the context of quantum integrable systems $[25,26]$ as the model-independent functional relations for eigenvalues of quantum transfer matrices. Moreover, some typical soliton equations can be obtained by performing a scaling continuum limit for appropriate combinations of parameters and variables, such as continuous Korteweg-de Vries (KdV) equation, KP equation, two-dimensional Toda lattice (2DTL) equation, Sine-Gordon (SG) equation, and Benjamin-Ono equation.

3.1. The Solutions in Terms of One-Theta Function. Suppose that

$$
\begin{aligned}
\zeta_{n+(a / 2), l+(b / 2)}^{m+(c / 2)} & =\Theta_{i}\left(k_{1} n+k_{2} l+k_{3} m+\frac{k_{1} a+k_{2} b+k_{3} c}{2}, \tau\right) \\
& =\Theta_{i}(\xi, \tau), \quad i=1,2,3,4,
\end{aligned}
$$

where

$$
\xi=k_{1} n+k_{2} l+k_{3} m+\frac{k_{1} a+k_{2} b+k_{3} c}{2},
$$

$k_{1}, k_{2}$, and $k_{3}$ are arbitrary constants, and $\Theta_{i}(\xi, \tau)$ is defined by (12). Similarly, we can obtain

$$
\begin{array}{ll}
\zeta_{n+a, l}^{m}=\Theta_{i}(\xi-\alpha, \tau), & \zeta_{n, l+b}^{m+c}=\Theta_{i}(\xi+\alpha, \tau), \\
\zeta_{n, l+b}^{m}=\Theta_{i}(\xi-\beta, \tau), & \zeta_{n+a, l}^{m+c}=\Theta_{i}(\xi+\beta, \tau), \\
\zeta_{n, l}^{m+c}=\Theta_{i}(\xi-\gamma, \tau), & \zeta_{n+a, l+b}^{m}=\Theta_{i}(\xi+\gamma, \tau),
\end{array}
$$

with

$$
\begin{gathered}
\alpha=\frac{k_{2} b+k_{3} c-k_{1} a}{2}, \quad \beta=\frac{k_{1} a+k_{3} c-k_{2} b}{2}, \\
\gamma=\frac{k_{1} a+k_{2} b-k_{3} c}{2} .
\end{gathered}
$$

Substituting (34) into (31) and using the following identities of the theta functions

$$
\begin{aligned}
\Theta_{1}(x & +y) \Theta_{1}(x-y) \\
& =\Theta_{2}^{-2}(0)\left[\Theta_{1}^{2}(x) \Theta_{2}^{2}(y)-\Theta_{2}^{2}(x) \Theta_{1}^{2}(y)\right], \\
\Theta_{2}(x+y) \Theta_{2}(x-y) & \\
& =\Theta_{2}^{-1}(0)\left[\Theta_{2}^{2}(x) \Theta_{2}^{2}(y)-\Theta_{1}^{2}(x) \Theta_{1}^{2}(y)\right], \\
\Theta_{3}(x & +y) \Theta_{3}(x-y) \\
& =\Theta_{2}^{-2}(0)\left[\Theta_{2}^{2}(x) \Theta_{3}^{2}(y)+\Theta_{1}^{2}(x) \Theta_{4}^{2}(y)\right], \\
\Theta_{4}(x & +y) \Theta_{4}(x-y) \\
& =\Theta_{2}^{-2}(0)\left[\Theta_{1}^{2}(x) \Theta_{3}^{2}(y)+\Theta_{2}^{2}(x) \Theta_{4}^{2}(y)\right],
\end{aligned}
$$

yield

$$
\begin{gathered}
a(b-c) \Theta_{2}^{2}(\alpha)+b(c-a) \Theta_{2}^{2}(\beta) \\
+c(a-b) \Theta_{2}^{2}(\gamma)=0, \\
a(b-c) \Theta_{1}^{2}(\alpha)+b(c-a) \\
\times \Theta_{1}^{2}(\beta)+c(a-b) \Theta_{1}^{2}(\gamma)=0, \\
\text { when } i=1,2 ; \\
a(b-c) \Theta_{3}^{2}(\alpha)+b(c-a) \Theta_{3}^{2}(\beta) \\
+c(a-b) \Theta_{3}^{2}(\gamma)=0, \\
a(b-c) \Theta_{4}^{2}(\alpha)+b(c-a) \Theta_{4}^{2}(\beta) \\
+c(a-b) \Theta_{4}^{2}(\gamma)=0 \\
\text { when } i=3,4 .
\end{gathered}
$$

Here, we use $\Theta_{i}(\xi)=\Theta_{i}(\xi, \tau)$ for simplicity. From (37), we can find that if $\alpha=\beta=\gamma$, that is, $k_{1} a=k_{2} b=k_{3} c$, which follows from (35), then (31) holds. In virtue of the variable 
transformation (30), the solution of (28) is expressed by onetheta function as follows:

$$
\begin{aligned}
u=\left(\Theta_{i}\left[k_{1}\left(n+\frac{a}{b} l+\frac{a}{c} m\right)\right]\right. & \left.\times \Theta_{i}\left[k_{1}\left(n+\frac{a}{b} l+\frac{a}{c} m\right)+2 k_{1} a\right]\right) \\
\times & \left(\Theta_{i}^{2}\left[k_{1}\left(n+\frac{a}{b} l+\frac{a}{c} m\right)+k_{1} a\right]\right)^{-1}-1, \\
v=1- & \left(\Theta_{i}\left[k_{1}\left(n+\frac{a}{b} l+\frac{a}{c} m\right)\right]\right. \\
\times & \times \Theta_{i}\left[\Theta_{1}\left(n+\frac{a}{b} l+\frac{a}{c} m\right)+2 k_{1} a\right] \\
& \left.\times \Theta_{i}\left[k_{1}\left(n+\frac{a}{b} l+\frac{a}{c} m\right)+3 k_{1} a\right]\right)
\end{aligned}
$$

Remark 3 (see [27]). If $\zeta_{n, l}^{m}=\Theta_{i}\left(\xi-\left(3 k_{1} a / 2\right), \tau\right)$ is a solution of (31), then $R_{0}((n / a)+(l / b)+(m / c)) R_{1}(n / a) R_{2}(l / b) R_{3}(m /$ c) $\Theta_{i}\left(\xi-\left(3 k_{1} a / 2\right), \tau\right)$ is a solution too, where $R_{i},(i=0,1,2,3)$, are arbitrary functions.

Remark 4 (see [27]). By means of the transformation, $\zeta_{n, l}^{m}=$ $[(b(c-a)) /(c(a-b))]^{(n l / a b)}[(b(c-a)) /(a(b-c))]^{(l m / b c)} \zeta_{n, l}^{m}$; then, $[(b(c-a)) /(c(a-b))]^{(n l / a b)}[(b(c-a)) /(a(b-c))]^{(l m / b c)} \Theta_{i}(\xi-$ $\left.\left(3 k_{1} a / 2\right), \tau\right)$ satisfies bilinear equation

$$
\zeta_{n+a, l}^{m} \zeta_{n, l+b}^{m+c}+\zeta_{n, l+b}^{m} \zeta_{n+a, l}^{m+c}+\zeta_{n, l}^{m+c} \zeta_{n+a, l+b}^{m}=0
$$

Proposition 5. The Riemann theta function $\Theta_{3}(\xi, \tau)$ defined by (12) has the periodic properties

$$
\Theta_{3}(\xi+1+\tau, \tau)=\exp (-2 i \pi \xi-i \pi \tau) \Theta_{3}(\xi, \tau)
$$

So, the meromorphic functions $F(\xi, \tau)$ defined by

$$
\begin{aligned}
& \text { (I) } F(\xi, \tau)=\frac{\Theta_{3}(\xi, \tau) \Theta_{3}(\xi+2 h, \tau)}{\Theta_{3}^{2}(\xi+h, \tau)}, \quad(\forall h, \xi \in C) \\
& \text { (II) } F(\xi, \tau)=\frac{\Theta_{3}(\xi, \tau) \Theta_{3}(\xi+3 h, \tau)}{\Theta_{3}(\xi+2 h, \tau) \Theta_{3}(\xi+h, \tau)}, \quad(\forall h, \xi \in C)
\end{aligned}
$$

are all double periodic functions with two fundamental periods 1 and $\tau$. Because the other three Riemann theta functions in (12) are the deformations of $\Theta_{3}(\xi, \tau)$, they can be proved to be periodic in a similar way.
Proof. By using definition $\Theta_{3}(\xi, \tau)$ in (12), it is easy to see that

$$
\begin{aligned}
\Theta_{3}(\xi+1+\tau, \tau) & =\sum_{m=-\infty}^{\infty} e^{2 m \pi i(\xi+1+\tau)+\pi i \tau m^{2}} \\
& =e^{-2 \pi i \xi} e^{-\pi i \tau} \Theta_{3}(\xi, \tau), \\
\Theta_{3}(\xi+h+1+\tau, \tau) & =\sum_{m=-\infty}^{\infty} e^{2 m \pi i(\xi+h+1+\tau)+\pi i \tau m^{2}} \\
& =e^{-2 \pi i(\xi+h)} e^{-\pi i \tau} \Theta_{3}(\xi+h, \tau) .
\end{aligned}
$$

Therefore, in both (I) and (II) cases, it holds that

$$
F(\xi+1+\tau, \tau)=F(\xi, \tau), \quad \xi \in C .
$$

That is to say, (38) is periodic wave solution of (28).

3.2. The Solutions in Terms of Two-Theta Function. In order to look for its solutions in terms of two-theta functions, we suppose

$$
\begin{aligned}
& \zeta_{n+(a / 2), l+(b / 2)}^{m+(c / 2)}= \Theta_{i}\left(\xi, \tau_{1}\right) \Theta_{j}\left(\xi, \tau_{2}\right) \\
&= \Theta_{i}\left(k_{1} n+k_{2} l+k_{3} m\right. \\
&\left.+\frac{k_{1} a+k_{2} b+k_{3} c}{2}, \tau_{1}\right) \\
& \times \Theta_{j}\left(k_{1} n+k_{2} l+k_{3} m\right. \\
&\left.+\frac{k_{1} a+k_{2} b+k_{3} c}{2}, \tau_{2}\right), \\
& \forall i, j=1,2,3,4 .
\end{aligned}
$$

Therefore,

$$
\begin{aligned}
& \zeta_{n+a, l}^{m}=\Theta_{i}\left(\xi-\alpha, \tau_{1}\right) \Theta_{j}\left(\xi-\alpha, \tau_{2}\right), \\
& \zeta_{n, l+b}^{m+c}=\Theta_{i}\left(\xi+\alpha, \tau_{1}\right) \Theta_{j}\left(\xi+\alpha, \tau_{2}\right), \\
& \zeta_{n, l+b}^{m}=\Theta_{i}\left(\xi-\beta, \tau_{1}\right) \Theta_{j}\left(\xi-\beta, \tau_{2}\right), \\
& \zeta_{n+a, l}^{m+c}=\Theta_{i}\left(\xi+\beta, \tau_{1}\right) \Theta_{j}\left(\xi+\beta, \tau_{2}\right), \\
& \zeta_{n, l}^{m+c}=\Theta_{i}\left(\xi-\gamma, \tau_{1}\right) \Theta_{j}\left(\xi-\gamma, \tau_{2}\right), \\
& \zeta_{n+a, l+b}^{m}=\Theta_{i}\left(\xi+\gamma, \tau_{1}\right) \Theta_{j}\left(\xi+\gamma, \tau_{2}\right),
\end{aligned}
$$

where $\alpha, \beta$, and $\gamma$ are the same as in (35).

Case 1. If $i=1, j=2$, we substitute (45) into (31) to get

$$
\begin{aligned}
a(b-c) \Theta_{1}\left(\xi-\alpha, \tau_{1}\right) \Theta_{2}\left(\xi-\alpha, \tau_{2}\right) \\
\quad \times \Theta_{1}\left(\xi+\alpha, \tau_{1}\right) \Theta_{2}\left(\xi+\alpha, \tau_{2}\right)+b(c-a) \\
\quad \times \Theta_{1}\left(\xi-\beta, \tau_{1}\right) \Theta_{2}\left(\xi-\beta, \tau_{2}\right) \Theta_{1}\left(\xi+\beta, \tau_{1}\right) \\
\quad \times \Theta_{2}\left(\xi+\beta, \tau_{2}\right)+c(a-b) \Theta_{1}\left(\xi-\gamma, \tau_{1}\right) \\
\quad \times \Theta_{2}\left(\xi-\gamma, \tau_{2}\right) \Theta_{1}\left(\xi+\gamma, \tau_{1}\right) \Theta_{2}\left(\xi+\gamma, \tau_{2}\right)=0 .
\end{aligned}
$$


By using the first two identities of the theta functions (36), the above equation is equivalent to

$$
\begin{gathered}
\Theta_{1}^{2}\left(\xi, \tau_{1}\right) \Theta_{2}^{2}\left(\xi, \tau_{2}\right)\left[\epsilon_{1} \Theta_{2}^{2}\left(\alpha, \tau_{1}\right) \Theta_{2}^{2}\left(\alpha, \tau_{2}\right)\right. \\
+\epsilon_{2} \Theta_{2}^{2}\left(\beta, \tau_{1}\right) \Theta_{2}^{2}\left(\beta, \tau_{2}\right) \\
\left.+\epsilon_{3} \Theta_{2}^{2}\left(\gamma, \tau_{1}\right) \Theta_{2}^{2}\left(\gamma, \tau_{2}\right)\right] \\
-\Theta_{1}^{2}\left(\xi, \tau_{1}\right) \Theta_{1}^{2}\left(\xi, \tau_{2}\right)\left[\epsilon_{1} \Theta_{2}^{2}\left(\alpha, \tau_{1}\right) \Theta_{1}^{2}\left(\alpha, \tau_{2}\right)\right. \\
+\epsilon_{2} \Theta_{2}^{2}\left(\beta, \tau_{1}\right) \Theta_{1}^{2}\left(\beta, \tau_{2}\right) \\
\left.+\epsilon_{3} \Theta_{2}^{2}\left(\gamma, \tau_{1}\right) \Theta_{1}^{2}\left(\gamma, \tau_{2}\right)\right] \\
-\Theta_{2}^{2}\left(\xi, \tau_{1}\right) \Theta_{2}^{2}\left(\xi, \tau_{2}\right)\left[\epsilon_{1} \Theta_{1}^{2}\left(\alpha, \tau_{1}\right) \Theta_{2}^{2}\left(\alpha, \tau_{2}\right)\right. \\
+\epsilon_{2} \Theta_{1}^{2}\left(\beta, \tau_{1}\right) \Theta_{2}^{2}\left(\beta, \tau_{2}\right) \\
\left.+\epsilon_{3} \Theta_{1}^{2}\left(\alpha, \tau_{1}\right) \Theta_{2}^{2}\left(\alpha, \tau_{2}\right)\right] \\
+\varepsilon_{1} \Theta_{1}^{2}\left(\alpha, \tau_{1}\right) \Theta_{1}^{2}\left(\alpha, \tau_{2}\right) \\
+\epsilon_{2} \Theta_{1}^{2}\left(\beta, \tau_{1}\right) \Theta_{1}^{2}\left(\beta, \tau_{2}\right) \\
\left.+\epsilon_{3} \Theta_{1}^{2}\left(\gamma, \tau_{1}\right) \Theta_{1}^{2}\left(\gamma, \tau_{2}\right)\right]=0
\end{gathered}
$$

Therefore,

$$
\begin{aligned}
& \epsilon_{1} \Theta_{2}^{2}\left(\alpha, \tau_{1}\right) \Theta_{2}^{2}\left(\alpha, \tau_{2}\right)+\epsilon_{2} \Theta_{2}^{2}\left(\beta, \tau_{1}\right) \Theta_{2}^{2}\left(\beta, \tau_{2}\right) \\
& \quad+\epsilon_{3} \Theta_{2}^{2}\left(\gamma, \tau_{1}\right) \Theta_{2}^{2}\left(\gamma, \tau_{2}\right)=0, \\
& \epsilon_{1} \Theta_{2}^{2}\left(\alpha, \tau_{1}\right) \Theta_{1}^{2}\left(\alpha, \tau_{2}\right)+\epsilon_{2} \Theta_{2}^{2}\left(\beta, \tau_{1}\right) \Theta_{1}^{2}\left(\beta, \tau_{2}\right) \\
& \quad+\epsilon_{3} \Theta_{2}^{2}\left(\gamma, \tau_{1}\right) \Theta_{1}^{2}\left(\gamma, \tau_{2}\right)=0, \\
& \epsilon_{1} \Theta_{1}^{2}\left(\alpha, \tau_{1}\right) \Theta_{2}^{2}\left(\alpha, \tau_{2}\right)+\epsilon_{2} \Theta_{1}^{2}\left(\beta, \tau_{1}\right) \Theta_{2}^{2}\left(\beta, \tau_{2}\right) \\
& \quad+\epsilon_{3} \Theta_{1}^{2}\left(\gamma, \tau_{1}\right) \Theta_{2}^{2}\left(\gamma, \tau_{2}\right)=0, \\
& \epsilon_{1} \Theta_{1}^{2}\left(\alpha, \tau_{1}\right) \Theta_{1}^{2}\left(\alpha, \tau_{2}\right)+\epsilon_{2} \Theta_{1}^{2}\left(\beta, \tau_{1}\right) \Theta_{1}^{2}\left(\beta, \tau_{2}\right) \\
& \quad+\epsilon_{3} \Theta_{1}^{2}\left(\gamma, \tau_{1}\right) \Theta_{1}^{2}\left(\gamma, \tau_{2}\right)=0 .
\end{aligned}
$$

Here, $\epsilon_{1}=a(b-c), \epsilon_{2}=b(c-a)$, and $\epsilon_{3}=c(a-b)$. To get special solutions of (48), we can assume that $\alpha=\beta=\gamma$; that is, $k_{1} a=k_{2} b=k_{3} c$. From the variable transformation (30), the solution of (28) in terms of two-theta functions are

$$
\begin{aligned}
u=\left(\Theta_{1}\left[k_{1}\left(n+\frac{a}{b} l+\frac{a}{c} m\right), \tau_{1}\right]\right. \\
\times \Theta_{2}\left[k_{1}\left(n+\frac{a}{b} l+\frac{a}{c} m\right), \tau_{2}\right] \\
\times \Theta_{1}\left[k_{1}\left(n+\frac{a}{b} l+\frac{a}{c} m\right)+2 k_{1} a, \tau_{1}\right] \\
\left.\times \Theta_{2}\left[k_{1}\left(n+\frac{a}{b} l+\frac{a}{c} m\right)+2 k_{1} a, \tau_{2}\right]\right)
\end{aligned}
$$

$$
\begin{gathered}
\times\left(\Theta_{1}^{2}\left[k_{1}\left(n+\frac{a}{b} l+\frac{a}{c} m\right)+k_{1} a, \tau_{1}\right]\right. \\
\left.\times \Theta_{2}^{2}\left[k_{1}\left(n+\frac{a}{b} l+\frac{a}{c} m\right)+k_{1} a, \tau_{2}\right]\right)^{-1}-1, \\
v=1-\left(\Theta_{1}\left[k_{1}\left(n+\frac{a}{b} l+\frac{a}{c} m\right), \tau_{1}\right]\right. \\
\times \Theta_{2}\left[k_{1}\left(n+\frac{a}{b} l+\frac{a}{c} m\right), \tau_{2}\right] \\
\times \Theta_{1}\left[k_{1}\left(n+\frac{a}{b} l+\frac{a}{c} m\right)+3 k_{1} a, \tau_{1}\right] \\
\left.\times \Theta_{2}\left[k_{1}\left(n+\frac{a}{b} l+\frac{a}{c} m\right)+3 k_{1} a, \tau_{2}\right]\right) \\
\times\left(\Theta_{1}\left[k_{1}\left(n+\frac{a}{b} l+\frac{a}{c} m\right)+2 k_{1} a, \tau_{1}\right]\right. \\
\times \Theta_{2}\left[k_{1}\left(n+\frac{a}{b} l+\frac{a}{c} m\right)+2 k_{1} a, \tau_{2}\right] \\
\times \Theta_{1}\left[k_{1}\left(n+\frac{a}{b} l+\frac{a}{c} m\right)+k_{1} a, \tau_{1}\right] \\
\left.\times \Theta_{2}\left[k_{1}\left(n+\frac{a}{b} l+\frac{a}{c} m\right)+k_{1} a, \tau_{2}\right]\right) .
\end{gathered}
$$

Case 2. If $i=2, j=3$ and substituting (45) into (31), then we are led to

$$
\begin{aligned}
a(b-c) \Theta_{2}\left(\xi-\alpha, \tau_{1}\right) \Theta_{3}\left(\xi-\alpha, \tau_{2}\right) \Theta_{2}\left(\xi+\alpha, \tau_{1}\right) \\
\quad \times \Theta_{3}\left(\xi+\alpha, \tau_{2}\right)+b(c-a) \Theta_{2}\left(\xi-\beta, \tau_{1}\right) \\
\quad \times \Theta_{3}\left(\xi-\beta, \tau_{2}\right) \Theta_{2}\left(\xi+\beta, \tau_{1}\right) \Theta_{3}\left(\xi+\beta, \tau_{2}\right) \\
\quad+c(a-b) \Theta_{2}\left(\xi-\gamma, \tau_{1}\right) \Theta_{3}\left(\xi-\gamma, \tau_{2}\right) \\
\quad \times \Theta_{2}\left(\xi+\gamma, \tau_{1}\right) \Theta_{3}\left(\xi+\gamma, \tau_{2}\right)=0 .
\end{aligned}
$$

From the identities of the theta functions (36), the last equation is changed into

$$
\begin{array}{r}
\Theta_{2}^{2}\left(\xi, \tau_{1}\right) \Theta_{2}^{2}\left(\xi, \tau_{2}\right)\left[\epsilon_{1} \Theta_{2}^{2}\left(\alpha, \tau_{1}\right) \Theta_{3}^{2}\left(\alpha, \tau_{2}\right)\right. \\
+\epsilon_{2} \Theta_{2}^{2}\left(\beta, \tau_{1}\right) \Theta_{3}^{2}\left(\beta, \tau_{2}\right) \\
\left.+\epsilon_{3} \Theta_{2}^{2}\left(\gamma, \tau_{1}\right) \Theta_{3}^{2}\left(\gamma, \tau_{2}\right)\right] \\
-\Theta_{1}^{2}\left(\xi, \tau_{1}\right) \Theta_{2}^{2}\left(\xi, \tau_{2}\right)\left[\epsilon_{1} \Theta_{1}^{2}\left(\alpha, \tau_{1}\right) \Theta_{3}^{2}\left(\alpha, \tau_{2}\right)\right. \\
+\epsilon_{2} \Theta_{1}^{2}\left(\beta, \tau_{1}\right) \Theta_{3}^{2}\left(\beta, \tau_{2}\right) \\
\left.+\epsilon_{3} \Theta_{1}^{2}\left(\gamma, \tau_{1}\right) \Theta_{3}^{2}\left(\gamma, \tau_{2}\right)\right] \\
+\Theta_{2}^{2}\left(\xi, \tau_{1}\right) \Theta_{1}^{2}\left(\xi, \tau_{2}\right)\left[\epsilon_{1} \Theta_{2}^{2}\left(\alpha, \tau_{1}\right) \Theta_{4}^{2}\left(\alpha, \tau_{2}\right)\right. \\
+\epsilon_{2} \Theta_{2}^{2}\left(\beta, \tau_{1}\right) \Theta_{4}^{2}\left(\beta, \tau_{2}\right) \\
\left.+\epsilon_{3} \Theta_{2}^{2}\left(\alpha, \tau_{1}\right) \Theta_{4}^{2}\left(\alpha, \tau_{2}\right)\right]
\end{array}
$$




$$
\begin{aligned}
-\Theta_{1}^{2}\left(\xi, \tau_{1}\right) \Theta_{1}^{2}\left(\xi, \tau_{2}\right)[ & \epsilon_{1} \Theta_{1}^{2}\left(\alpha, \tau_{1}\right) \Theta_{4}^{2}\left(\alpha, \tau_{2}\right) \\
& +\epsilon_{2} \Theta_{1}^{2}\left(\beta, \tau_{1}\right) \Theta_{4}^{2}\left(\beta, \tau_{2}\right) \\
& \left.+\epsilon_{3} \Theta_{1}^{2}\left(\gamma, \tau_{1}\right) \Theta_{4}^{2}\left(\gamma, \tau_{2}\right)\right]=0 .
\end{aligned}
$$

Therefore,

$$
\begin{aligned}
& \epsilon_{1} \Theta_{2}^{2}\left(\alpha, \tau_{1}\right) \Theta_{3}^{2}\left(\alpha, \tau_{2}\right)+\epsilon_{2} \Theta_{2}^{2}\left(\beta, \tau_{1}\right) \Theta_{3}^{2}\left(\beta, \tau_{2}\right) \\
& \quad+\epsilon_{3} \Theta_{2}^{2}\left(\gamma, \tau_{1}\right) \Theta_{3}^{2}\left(\gamma, \tau_{2}\right)=0, \\
& \epsilon_{1} \Theta_{1}^{2}\left(\alpha, \tau_{1}\right) \Theta_{3}^{2}\left(\alpha, \tau_{2}\right)+\epsilon_{2} \Theta_{1}^{2}\left(\beta, \tau_{1}\right) \Theta_{3}^{2}\left(\beta, \tau_{2}\right) \\
& \quad+\epsilon_{3} \Theta_{1}^{2}\left(\gamma, \tau_{1}\right) \Theta_{3}^{2}\left(\gamma, \tau_{2}\right)=0, \\
& \epsilon_{1} \Theta_{2}^{2}\left(\alpha, \tau_{1}\right) \Theta_{4}^{2}\left(\alpha, \tau_{2}\right)+\epsilon_{2} \Theta_{2}^{2}\left(\beta, \tau_{1}\right) \Theta_{4}^{2}\left(\beta, \tau_{2}\right) \\
& \quad+\epsilon_{3} \Theta_{2}^{2}\left(\alpha, \tau_{1}\right) \Theta_{4}^{2}\left(\alpha, \tau_{2}\right)=0, \\
& \epsilon_{1} \Theta_{1}^{2}\left(\alpha, \tau_{1}\right) \Theta_{4}^{2}\left(\alpha, \tau_{2}\right)+\epsilon_{2} \Theta_{1}^{2}\left(\beta, \tau_{1}\right) \Theta_{4}^{2}\left(\beta, \tau_{2}\right) \\
& \quad+\epsilon_{3} \Theta_{1}^{2}\left(\gamma, \tau_{1}\right) \Theta_{4}^{2}\left(\gamma, \tau_{2}\right)=0,
\end{aligned}
$$

with $\epsilon_{1}=a(b-c), \epsilon_{2}=b(c-a)$, and $\epsilon_{3}=c(a-b)$. To get the special solutions of (52), we can assume that $\alpha=\beta=\gamma$; that is, $k_{1} a=k_{2} b=k_{3} c$. From the variable transformation (30), the solution of (28) in terms of two-theta functions are

$$
\begin{aligned}
& u=\left(\Theta_{2}[\right.\left.k_{1}\left(n+\frac{a}{b} l+\frac{a}{c} m\right), \tau_{1}\right] \\
& \times \Theta_{3}\left[k_{1}\left(n+\frac{a}{b} l+\frac{a}{c} m\right), \tau_{2}\right] \\
& \times \Theta_{2}\left[k_{1}\left(n+\frac{a}{b} l+\frac{a}{c} m\right)+2 k_{1} a, \tau_{1}\right] \\
&\left.\times \Theta_{3}\left[k_{1}\left(n+\frac{a}{b} l+\frac{a}{c} m\right)+2 k_{1} a, \tau_{2}\right]\right) \\
& \times\left(\Theta_{2}^{2}\left[k_{1}\left(n+\frac{a}{b} l+\frac{a}{c} m\right)+k_{1} a, \tau_{1}\right]\right. \\
&\left.\times \Theta_{3}^{2}\left[k_{1}\left(n+\frac{a}{b} l+\frac{a}{c} m\right)+k_{1} a, \tau_{2}\right]\right)^{-1}-1, \\
& v=\left(\Theta_{2}\left[k_{1}\left(n+\frac{a}{b} l+\frac{a}{c} m\right), \tau_{1}\right]\right. \\
& \times \Theta_{3}\left[k_{1}\left(n+\frac{a}{b} l+\frac{a}{c} m\right), \tau_{2}\right] \\
& \times \Theta_{2}\left[k_{1}\left(n+\frac{a}{b} l+\frac{a}{c} m\right)+3 k_{1} a, \tau_{1}\right] \\
&\left.\times \Theta_{3}\left[k_{1}\left(n+\frac{a}{b} l+\frac{a}{c} m\right)+3 k_{1} a, \tau_{2}\right]\right)
\end{aligned}
$$

$$
\begin{aligned}
\times \Theta_{2}( & {\left[k_{1}\left(n+\frac{a}{b} l+\frac{a}{c} m\right)+2 k_{1} a, \tau_{1}\right] } \\
& \times \Theta_{3}\left[k_{1}\left(n+\frac{a}{b} l+\frac{a}{c} m\right)+2 k_{1} a, \tau_{2}\right] \\
& \times \Theta_{2}\left[k_{1}\left(n+\frac{a}{b} l+\frac{a}{c} m\right)+k_{1} a, \tau_{1}\right] \\
& \left.\times \Theta_{3}\left[k_{1}\left(n+\frac{a}{b} l+\frac{a}{c} m\right)+k_{1} a, \tau_{2}\right]\right)^{-1} .
\end{aligned}
$$

Proposition 6. The Riemann theta functions $\Theta_{2}(\xi, \tau)$, $\Theta_{3}(\xi, \tau)$ defined by (12) have the periodic properties

$$
\begin{aligned}
& \Theta_{2}(\xi+2+\tau, \tau)=\exp (-2 i \pi \xi-i \pi \tau) \Theta_{2}(\xi, \tau), \\
& \Theta_{3}(\xi+1+\tau, \tau)=\exp (-2 i \pi \xi-i \pi \tau) \Theta_{3}(\xi, \tau) .
\end{aligned}
$$

So, the meromorphic functions $F_{1}(\xi, \tau), F_{2}(\xi, \tau)$ defined by

$$
\begin{aligned}
& F_{1}(\xi, \tau)=\frac{\Theta_{2}(\xi, \tau) \Theta_{2}(\xi+2 h, \tau)}{\Theta_{2}^{2}(\xi+h, \tau)}, \\
& F_{2}(\xi, \tau)=\frac{\Theta_{3}(\xi, \tau) \Theta_{3}(\xi+3 h, \tau)}{\Theta_{3}(\xi+2 h, \tau) \Theta_{3}(\xi+h, \tau)}
\end{aligned}
$$

$(\forall h, \xi \in C)$

are all periodic functions; moreover, $F_{1}(\xi, \tau) * F_{2}(\xi, \tau)$ is also periodic functions.

From Proposition 6, we verify that (53) is periodic wave solution of (28). In a similar way, the other cases of two-theta functions solutions of (28) can also be proved to be periodic.

\section{Conclusion}

In this paper, based on the Riemann theta functions, a lucid and straightforward generalization of the Hirota-Riemann method is presented to explicitly construct some kinds of quasiperiodic wave solutions for $(3+1)$-dimensional potential-YTSF equation and discrete KP equation. This method is also suitable for other more general nonlinear evolution equations in mathematical physics. Moreover, for the $(3+1)$-dimensional potential-YTSF equation, we not only use linear superposition principle to generate $\mathrm{N}$-wave solutions but also analyze the quasiperiodic wave solutions that tend to the soliton solutions under a small amplitude limit.

\section{Acknowledgments}

This work was partially supported by the National Natural Science Foundation of China (Grant no. 11271246), the Natural Science Research Project of Henan Education Department (Grant no. 2011B110024), and the Research Fund for Luoyang Normal University (no. qnjj-2009-02).

\section{References}

[1] M. J. Ablowitz and H. Segur, Solitons and the Inverse Scattering Transform, Cambridge University Press, Cambridge, UK, 2006. 
[2] R. Hirota, Direct Methods in Soliton Theory, Cambridge University Press, Cambridge, UK; Springer, New York, NY, USA, 2004.

[3] Y. T. Wu and X. B. Hu, "A new integrable differential-difference system and its explicit solutions," Journal of Physics A, vol. 32, no. 8, p. 1515, 1999.

[4] X. G. Geng and H. H. Dai, "Quasi-periodic solutions for some 2 + 1-dimensional discrete models," Physica A, vol. 319, no. 1-4, pp. 270-294, 2003.

[5] C. W. Cao, X. G. Geng, and H. Wang, "Algebro-geometric solution of the $2+1$ dimensional Burgers equation with a discrete variable," Journal of Mathematical Physics, vol. 43, no. 1, pp. 621643, 2002.

[6] S. Xue and D. L. Du, "A new hierarchy of $(1+1)$-dimensional soliton equations and its quasi-periodic solutions," Chaos, Solitons and Fractals, vol. 35, no. 4, pp. 692-704, 2008.

[7] W. X. Ma, "An application of the casoratian technique to the $2 \mathrm{~d}$ toda lattice equation," Modern Physics Letters B, vol. 22, no. 19, p. 1815, 2008.

[8] J. M. Wang and X. Yang, "Quasi-periodic wave solutions for the $(2+1)$-dimensional generalized CalogeroBogoyavlenskiiSchiff (CBS) equation," Nonlinear Analysis, Theory, Methods and Applications, vol. 75, no. 4, pp. 2256-2261, 2012.

[9] A. Nakamura, "A direct method of calculating periodic wave solutions to nonlinear evolution equations. I. Exact twoperiodic wave solution," Journal of the Physical Society of Japan, vol. 47, no. 5, pp. 1701-1705, 1979.

[10] A. Nakamura, "A direct method of calculating periodic wave solutions to nonlinear evolution equations. II. Exact one- and two-periodic wave solution of the coupled bilinear e," Journal of the Physical Society of Japan, vol. 48, no. 4, pp. 1365-1370, 1980.

[11] E. G. Fan and Y. C. Hon, "Quasiperiodic waves and asymptotic behavior for Bogoyavlenskii's breaking soliton equation in $(2+$ 1) dimensions," Physical Review E, vol. 78, no. 3, Article ID 036607, 2008.

[12] E. G. Fan and K. W. Chow, "On the periodic solutions for both nonlinear differential and difference equations: a unified approach," Physics Letters A, vol. 374, no. 35, pp. 3629-3634, 2010.

[13] Z. J. Qiao and E. G. Fan, "Negative-order Korteweg-de Vries equations," Physical Review E, vol. 86, no. 1, Article ID 016601, 2012.

[14] Y. C. Hon and Q. Wang, "Construction of quasi-periodic wave solutions for differential-difference equation," Zeitschrift für Naturforschung A, vol. 67, p. 21, 2012.

[15] S.-F. Tian and H.-Q. Zhang, "A kind of explicit Riemann theta functions periodic waves solutions for discrete soliton equations," Communications in Nonlinear Science and Numerical Simulation, vol. 16, no. 1, pp. 173-186, 2011.

[16] S. J. Yu, K. Toda, N. Sasa, and T. Fukuyama, "N soliton solutions to the Bogoyavlenskii-Schiff equation and a quest for the soliton solution in $(3+1)$ dimensions," Journal of Physics A, vol. 31, no. 14, pp. 3337-3347, 1998.

[17] Z. Y. Yan, "New families of nontravelling wave solutions to a new (3+1)-dimensional potential-YTSF equation," Physics Letters A, vol. 318, no. 1-2, pp. 78-83, 2003.

[18] T. X. Zhang, H. N. Xuan, D. Zhang, and C. Wang, "Nontravelling wave solutions to a $(3+1)$-dimensional potentialYTSF equation and a simplified model for reacting mixtures," Chaos, Solitons and Fractals, vol. 34, no. 3, pp. 1006-1013, 2007.

[19] X. P. Zeng, Z. D. Dai, and D. Li, "New periodic soliton solutions for the $(3+1)$-dimensional potential-YTSF equation," Chaos, Solitons and Fractals, vol. 42, no. 2, pp. 657-661, 2009.
[20] M. Abramowitz and I. Stegun, Handbook of Mathematical Functions, Dover, New York, NY, USA, 1965.

[21] Y. Ohta, R. Hirota, S. Tsujimoto, and T. Imai, "Casorati and discrete gram type determinant representations of solutions to the discrete KP hierarchy," Journal of the Physical Society of Japan, vol. 62, pp. 1872-1886, 1993.

[22] R. Hirota, "Nonlinear partial difference equations. I. A difference analogue of the Korteweg-de Vries equation," vol. 43, no. 4, pp. 1424-1433, 1977.

[23] M. Kashiwara and T. Miwa, "Transformation groups for soliton equations. I. The $\tau$ function of the Kadomtsev-Petviashvili equation," Proceedings of the Japan Academy A, vol. 57, no. 7, pp. 342-347, 1981.

[24] T. Miwa, "On Hirota's difference equations," Proceedings of the Japan Academy A, vol. 58, no. 1, pp. 9-12, 1982.

[25] A. Zabrodin, "Discrete Hirota's equation in quantum integrable models," International Journal of Modern Physics B, vol. 11, no. 26-27, p. 3125, 1997.

[26] I. Krichever, O. Lipan, P. Wiegmann, and A. Zabrodin, "Quantum integrable models and discrete classical Hirota equations," Communications in Mathematical Physics, vol. 188, no. 2, pp. 267-304, 1997.

[27] A. V. Zabrodin, "Hirota difference equations," Theoretical and Mathematical Physics, vol. 113, no. 2, pp. 1347-1392, 1997. 


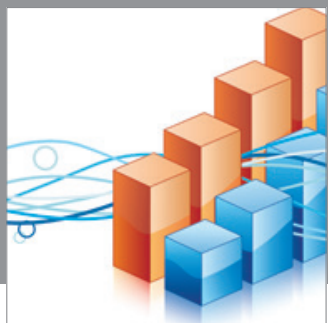

Advances in

Operations Research

mansans

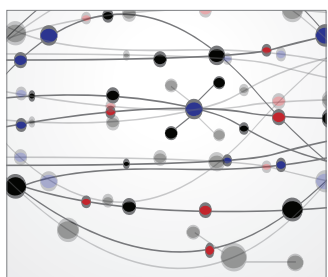

The Scientific World Journal
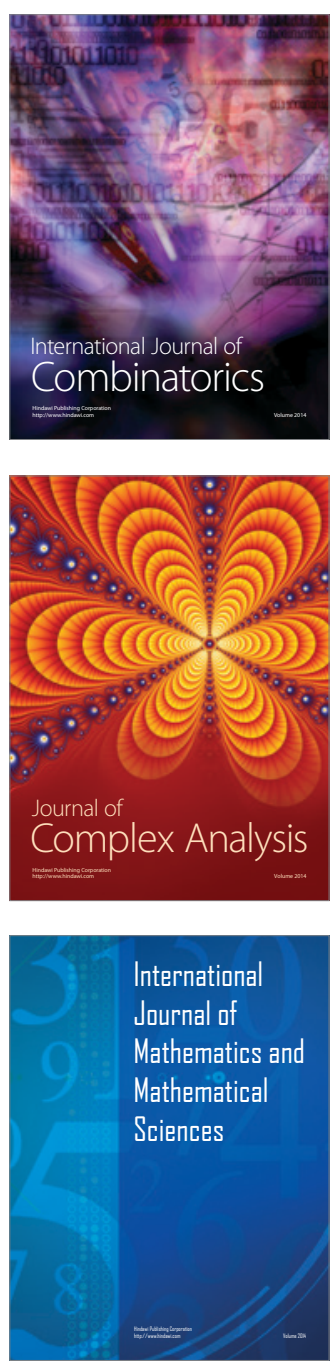
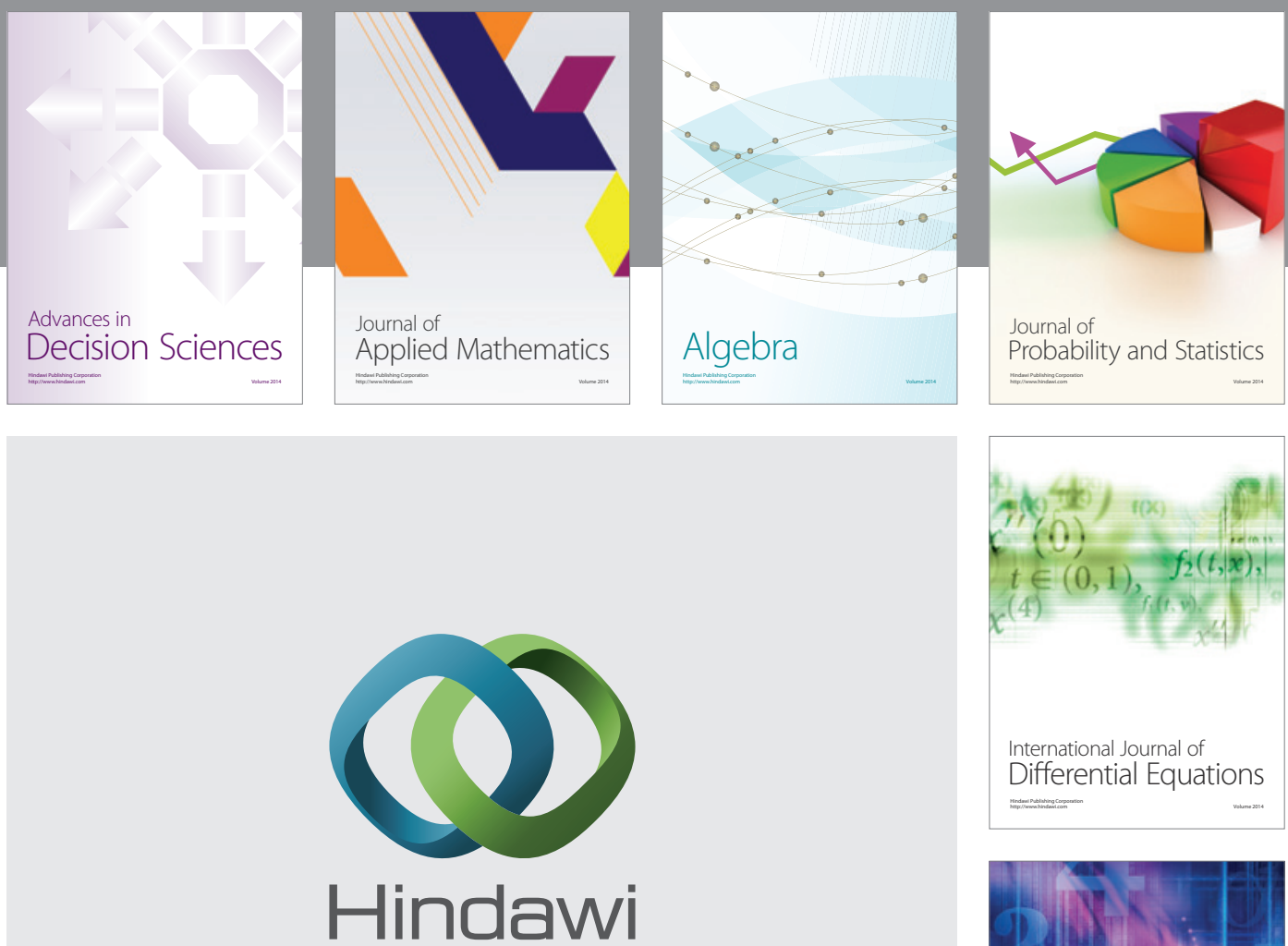

Submit your manuscripts at http://www.hindawi.com
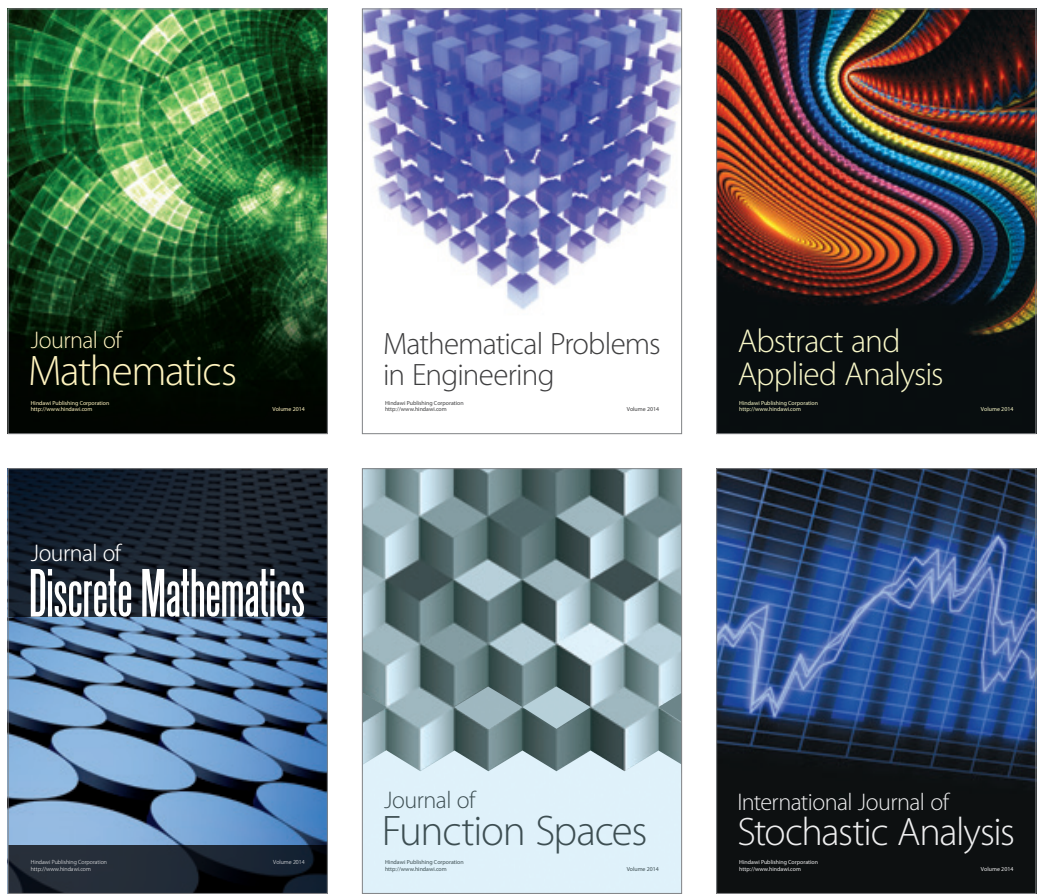

Journal of

Function Spaces

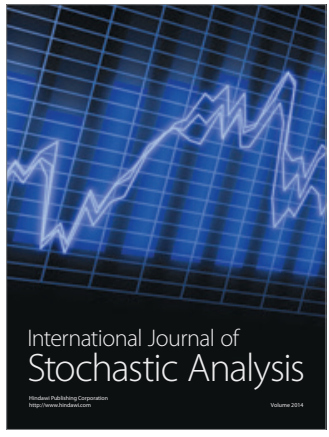

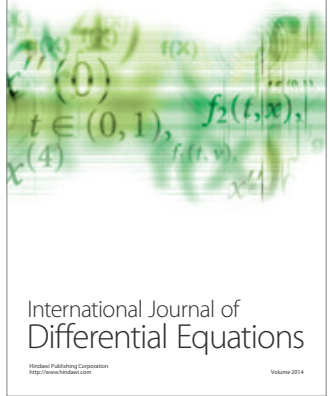
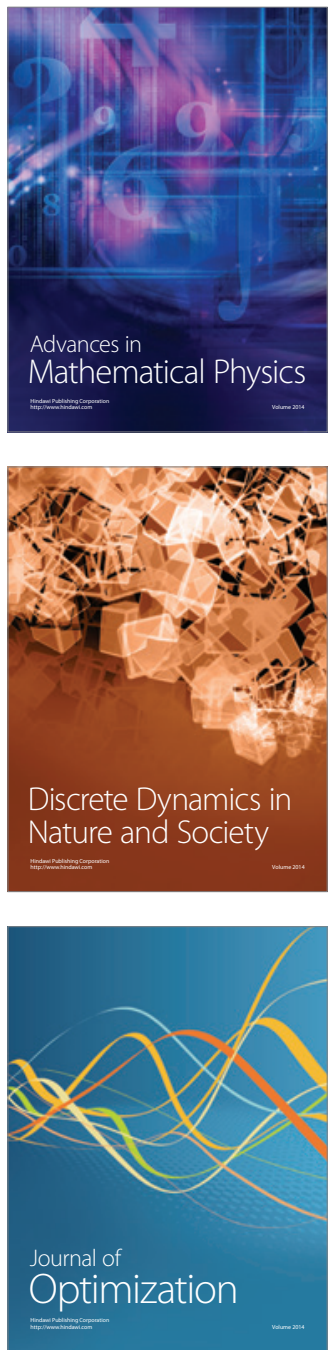